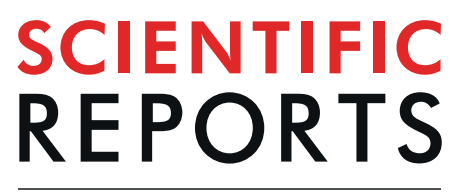

natureresearch

Check for updates

\title{
Incidence and risk of sepsis following appendectomy: a nationwide population-based cohort study
}

\begin{abstract}
Meng-Che Wu ${ }^{1,2}$, Hsi-Kai Tsou ${ }^{3,4}$, Cheng-Li Lin ${ }^{5,6}$ \& James Cheng-Chung Wei, $i^{7,9,10 凶 ~}$
Appendectomy is a frequently performed surgical procedure; however, long-term consequences have not been fully explored. We used a nationwide population-based cohort to determine whether patients undergoing appendectomy are at an increased risk of sepsis. Overall, 252,688 patients undergoing appendectomy and 252,472 matched controls were identified from the National Health Insurance Research Database in Taiwan. A propensity score analysis was used for matching age, sex, index year and comorbidities at a ratio of 1:1. Multiple Cox regression and stratified analyses were used to estimate the adjusted hazard ratio (aHR) of developing sepsis. Patients undergoing appendectomy had a 1.29 times (aHR: 1.29; 95\% confidence interval [Cl], 1.26-1.33) higher risk of developing sepsis than those not undergoing. Patients aged 20-49 years had a 1.58-fold higher risk of sepsis in the appendectomy cohort (aHR; $95 \% \mathrm{Cl}, 1.50-1.68)$. Also, having undergone appendectomy, patients had a higher likelihood of sepsis, regardless of sex and with or without comorbidities. Patients with $<1$ year follow-up showed a 1.98-fold risk of sepsis in the appendectomy cohort. Patients with 1-4 and $\geq 5$ years follow-up showed a 1.29 and 1.11-fold risk of sepsis, respectively. Future research is required to elucidate the possible immuno-pathological mechanisms of these associations.
\end{abstract}

Sepsis is a clinical heterogeneous syndrome that is defined as a life-threatening organ dysfunction caused by a dysregulated host response to an infection' ${ }^{1,2}$. Sepsis and the subsequent systemic inflammatory response can lead to multiple organ dysfunction and even death ${ }^{1}$. It remains a clinical challenge for clinicians and researchers.

Appendectomy is one of the most frequently performed emergent abdominal procedures worldwide. However, it has recently been recognised that the appendix is not just a vestigial structure of the gut, but rather is an important organ for the development and preservation of the gut immune system ${ }^{3,4}$. The human vermiform appendix provides a continuous source of commensal flora, thereby crowding out potentially unhealthy microorganisms, restoring diversity and stability of the gut microbiome and contributing to defensive immune mechanisms over a lifetime. Thus, it is known as a 'safe house' for normal gut flora. The appendix has been significantly associated with recurrent Clostridioides difficile infection ${ }^{4}$ and recurrence of small bowel bacterial overgrowth after antibiotics administration ${ }^{5}$. Studies have also examined the association between antecedent removal of the appendix and the risk of various diseases such as systemic lupus erythematosus ${ }^{6}$, rheumatoid arthritis ${ }^{7}$, inflammatory bowel disease $\mathrm{e}^{8}$ and cancers ${ }^{9-11}$. These conditions reflect the important role of the appendix in microbial ecology and intestinal mucosal immunity.

\footnotetext{
${ }^{1}$ Institute of Medicine, Chung Shan Medical University, Taichung, Taiwan. ${ }^{2}$ Division of Gastroenterology, Children's Medical Center, Taichung Veterans General Hospital, Taichung, Taiwan. ${ }^{3}$ Functional Neurosurgery Division, Neurological Institute, Taichung Veterans General Hospital, Taichung, Taiwan. ${ }^{4}$ Department of Rehabilitation, JenTeh Junior College of Medicine, Nursing and Management, Miaoli County, Taiwan. ${ }^{5}$ College of Medicine, China Medical University, Taichung, Taiwan. ${ }^{6}$ Management Office for Health Data, China Medical University Hospital, Taichung, Taiwan. ${ }^{7}$ Department of Rheumatology, BenO Medical Center, The Affiliated BenQ Hospital of Nanjing Medical University, Nanjing, China. ${ }^{8}$ Beijing Tsinghua Changgung Hospital, School of Clinical Medicine, Tsinghua University, Beijing, China. ${ }^{9}$ Division of Allergy, Immunology and Rheumatology, Chung Shan Medical University Hospital; Institute of Medicine, College of Medicine, Chung Shan Medical University, Taichung, Taiwan. ${ }^{10} \mathrm{Graduate}$ Institute of Integrated Medicine, China Medical University, Taichung, Taiwan. ${ }^{凶}$ e-mail: jccwei@gmail.com
} 


\begin{tabular}{|c|c|c|c|c|c|}
\hline & \multicolumn{4}{|c|}{ Appendectomy } & \multirow[b]{4}{*}{ Pvalue } \\
\hline & \multicolumn{2}{|c|}{ No } & \multicolumn{2}{|l|}{ Yes } & \\
\hline & \multicolumn{2}{|c|}{$\mathrm{N}=252,472$} & \multicolumn{2}{|c|}{$\mathrm{N}=252,688$} & \\
\hline & $\mathrm{n}$ & $\%$ & $\mathrm{n}$ & $\%$ & \\
\hline Age (years) & & & & & 0.97 \\
\hline $20-49$ & 179,762 & 71.2 & 179,847 & 71.2 & \\
\hline $50-64$ & 42,590 & 16.9 & 42,644 & 16.9 & \\
\hline$\geq 65$ & 30,120 & 11.9 & 30,197 & 12.0 & \\
\hline $\operatorname{Median}(\mathrm{SD})^{*}$ & 41.7 & 17.0 & 41.8 & 16.8 & 0.01 \\
\hline Sex & & & & & 0.97 \\
\hline Women & 122,127 & 48.4 & 122,218 & 48.4 & \\
\hline Men & 130,345 & 51.6 & 130,470 & 51.6 & \\
\hline \multicolumn{6}{|l|}{ Comorbidity } \\
\hline Diabetes mellitus & 12,884 & 5.10 & 12,991 & 5.14 & 0.54 \\
\hline Hypertension & 24,341 & 9.64 & 24,456 & 9.68 & 0.65 \\
\hline Hyperlipidemia & 5465 & 2.16 & 5558 & 2.20 & 0.40 \\
\hline Atrial fibrillation & 1486 & 0.59 & 1580 & 0.63 & 0.09 \\
\hline Stroke & 5671 & 2.25 & 5769 & 2.28 & 0.38 \\
\hline $\begin{array}{l}\text { Congestive heart } \\
\text { failure }\end{array}$ & 2193 & 0.87 & 2290 & 0.91 & 0.15 \\
\hline
\end{tabular}

Table 1. Baseline characteristics for individuals with and without appendectomy. Chi-square test; *MannWhitney U test; SD, standard deviation.

The short-term complications of appendectomy are well-studied, whereas only limited long-term consequences have been assessed ${ }^{12}$. The absence of the appendix might alter immune function and the gut microbiome. It is postulated that in susceptible populations, the intestinal epithelium becomes hyper-permeable and apoptotic during critical illness, which results in intestinal flora leaking into the systemic circulation and potentially leading to systemic inflammation and organ failure. The gut microbiota is also thought to play a pivotal role in the pathogenesis of sepsis ${ }^{13}$. It has been shown that the gut microbiome composition of patients with sepsis is profoundly distorted ${ }^{14}$. Thus, we hypothesised that antecedent appendectomy could impact the future risk of sepsis and evaluated this hypothesis by analysing a nationwide population-based retrospective cohort from the Taiwanese National Health Insurance Research Database (NHIRD).

\section{Results}

We identified 252,688 patients undergoing appendectomy and 252,472 matched controls between 2000 and 2013 from the National Health Insurance Research Database in Taiwan. The median follow-up periods for the appendectomy and non-appendectomy cohorts were 7.04 and 6.96 years, respectively. Table 1 shows the demographic characteristics of the patients. There were $71.2 \%, 16.9 \%$ and $11.9 \%$ of patients in the $20-49$ age group, $50-64$ age group and $\geq 65$ age group, respectively, and about $48.4 \%$ of participants were women and about $51.6 \%$ of patients were men. The median age in the exposed cohort was $41.8 \pm 16.8$ years, and the average age in the unexposed cohort was $41.7 \pm 17.0$ years. In terms of comorbidities, there were no statistically significant differences between the exposed cohort and the unexposed cohort.

Table 2 displays the incidence and risk factors for sepsis. The incidence rate was 6.05 per 1,000 person-years among the appendectomy cohort. After adjustment, patients with appendectomy had a significantly higher risk of developing sepsis than those without appendectomy (aHR, 1.29; 95\% CI, 1.26-1.33; P<0.001). Further analysis of the extended Cox models with time-dependent terms showed similar results, indicating that the strength of the association increased over time (HR [P-value] for appendectomy, $1.23[\mathrm{P}<0.001]$ and for the interaction term of appendectomy and time, $1.09[\mathrm{P}<0.001])$.

Older age groups were associated with a higher risk of developing sepsis when they were compared with the 20-49 age group (aHR, 3.59; 95\% CI, 3.45-3.75; $\mathrm{P}<0.001$ in the 50-64 age group \& aHR, 10.2; 95\% CI, 9.85-10.6; $\mathrm{P}<0.001$ in the $\geq 65$ age group). Compared with women, men had a higher risk of sepsis (aHR, 1.15; 95\% CI, 1.12-1.18; $\mathrm{P}<0.001$ ). In terms of comorbidities, patients with diabetes mellitus (aHR, 2.01; 95\% CI, 1.94-2.09; $\mathrm{P}<0.001$ ), hypertension (aHR, 1.48; 95\% CI, 1.42-1.53; $\mathrm{P}<0.001$ ), atrial fibrillation (aHR, 1.31; 95\% CI, 1.211.43; $\mathrm{P}<0.001$ ), stroke (aHR, 1.56; 95\% CI, 1.49-1.64; $\mathrm{P}<0.001)$ and congestive heart failure (aHR, 1.68; 95\% CI, $1.57-1.80 ; \mathrm{P}<0.001)$, were all at higher risk of sepsis.

In Table 3, stratified analyses were performed to assess the association between appendectomy and sepsis based on demographic characteristics. In patients aged 20-49 years, compared with the unexposed cohort, there was a 1.58-fold higher risk of sepsis in the exposed cohort (aHR; 95\% CI, 1.50-1.68; P $<0.001$ ). In patients aged 50-64 years, compared with the unexposed cohort, there was 1.40-fold higher risk of sepsis in the exposed cohort (aHR; 95\% CI, 1.32-1.48; $\mathrm{P}<0.001$ ). In patients aged $\geq 65$ years, compared with the unexposed cohort, there was a 1.13-fold higher risk of sepsis in the exposed cohort (aHR; 95\% CI, 1.09-1.18; P $<0.001$ ). Among women, compared with patients without appendectomy, there was a 1.39-fold higher risk of sepsis in patients with appendectomy (aHR; 95\% CI, 1.33-1.45; P<0.001). Among men, compared with patients without appendectomy, 


\begin{tabular}{|c|c|c|c|c|c|}
\hline & Event & PY & Rate $^{*}$ & $\begin{array}{l}\text { Crude HR } \\
(95 \% \text { CI })\end{array}$ & $\begin{array}{l}\text { Adjusted HR \& } \\
(95 \% \mathrm{CI})\end{array}$ \\
\hline \multicolumn{6}{|c|}{ Appendectomy } \\
\hline No & 8678 & $1,808,705$ & 4.80 & 1.00 & 1.00 \\
\hline Yes & 10847 & $1,791,553$ & 6.05 & $1.26(1.23,1.30)^{* * *}$ & $1.29(1.26,1.33)^{* * *}$ \\
\hline \multicolumn{6}{|l|}{ Age } \\
\hline $20-49$ & 5005 & $2,694,333$ & 1.86 & 1.00 & 1.00 \\
\hline $50-64$ & 4467 & 562,009 & 7.95 & $4.35(4.18,4.53)^{* * * *}$ & $3.59(1.25,1.33)^{* * *}$ \\
\hline$\geq 65$ & 10053 & 343,916 & 29.2 & $16.2(15.7,16.8)^{* * * *}$ & $10.2(9.85,10.6)^{* * *}$ \\
\hline \multicolumn{6}{|l|}{ Gender } \\
\hline Women & 8851 & $1,752,543$ & 5.05 & 1.00 & 1.00 \\
\hline Men & 10674 & $1,847,716$ & 5.78 & $1.14(1.11,1.18)^{* * *}$ & $1.15(1.12,1.18)^{* * * *}$ \\
\hline \multicolumn{6}{|c|}{ Comorbidity } \\
\hline \multicolumn{6}{|c|}{ Diabetes mellitus } \\
\hline No & 15,364 & $3,460,921$ & 4.44 & 1.00 & 1.00 \\
\hline Yes & 4161 & 139,337 & 29.9 & $6.82(6.59,7.06)^{* * *}$ & $2.01(1.94,2.09) * * *$ \\
\hline \multicolumn{6}{|c|}{ Hypertension } \\
\hline No & 12,917 & $3,343,842$ & 3.86 & 1.00 & 1.00 \\
\hline Yes & 6608 & 256,417 & 25.8 & $6.85(6.65,7.06)^{* * *}$ & $1.48(1.42,1.53)^{* * * *}$ \\
\hline \multicolumn{6}{|c|}{ Hyperlipidemia } \\
\hline No & 18,147 & $3,538,639$ & 5.13 & 1.00 & 1.00 \\
\hline Yes & 1378 & 61,619 & 22.4 & $4.38(4.15,4.63)^{* * * *}$ & $1.05(0.99,1.12)$ \\
\hline \multicolumn{6}{|c|}{ Atrial fibrillation } \\
\hline No & 18,898 & $3,586,432$ & 5.27 & 1.00 & 1.00 \\
\hline Yes & 627 & 13827 & 45.4 & $8.64(7.97,9.35)^{* * *}$ & $1.31(1.21,1.43)^{* * * *}$ \\
\hline \multicolumn{6}{|l|}{ Stroke } \\
\hline No & 17,221 & 3,541995 & 4.86 & 1.00 & 1.00 \\
\hline Yes & 2304 & 58,263 & 39.5 & $8.22(7.87,8.59) * * *$ & $1.56(1.49,1.64)^{* * * *}$ \\
\hline \multicolumn{6}{|c|}{ Congestive heart failure } \\
\hline No & 18,492 & $3,581,114$ & 5.16 & 1.00 & 1.00 \\
\hline Yes & 1033 & 19,144 & 54.0 & $10.5(9.86,11.2)^{* * *}$ & $1.68(1.57,1.80)^{* * *}$ \\
\hline
\end{tabular}

Table 2. The incidence of and risk factors for sepsis. CI confidence interval; HR, hazard ratio; PY, personyears; ${ }^{\#}$ Incidence rate per 1,000 person-years; ${ }^{8}$ Multivariable analysis including age, gender and comorbidities of diabetes mellitus, hypertension, hyperlipidemia, atrial fibrillation, stroke and congestive heart failure; *** $\mathrm{P}<0.001$

there was 1.22-fold higher risk of sepsis in patients with appendectomy (aHR; 95\% CI, 1.17-1.27; P $<0.001$ ). For patients without any comorbidity, compared with the unexposed cohort, there was 1.51-fold higher risk of sepsis in the exposed cohort (aHR; 95\% CI, 1.45-1.57; P $<0.001$ ). For patients with any comorbidity, compared with the unexposed cohort, there was a 1.10-fold higher risk of sepsis in the exposed cohort (aHR; 95\% CI, 1.06-1.15; $\mathrm{P}<0.001$ ). For patients whose years of follow-up were $<1$ year, there was a 1.98 -fold higher risk of sepsis in the exposed cohort when compared with the unexposed cohort (aHR; 95\% CI, 1.84-2.14; $\mathrm{P}<0.001$ ). For patients whose years of follow-up were 1-4 years, there was a 1.29-fold higher risk of sepsis in the exposed cohort when compared with the unexposed cohort (aHR; 95\% CI, 1.23-1.34; $\mathrm{P}<0.001$ ). For patients whose years of follow-up were $\geq 5$ years, there was a 1.11 -fold higher risk of sepsis in the exposed cohort when compared with the unexposed cohort $(\mathrm{aHR} ; 95 \% \mathrm{CI}, 1.06-1.16$; $\mathrm{P}<0.01)$. In Table 4, similar results were observed for sepsis through inverse probability of treatment weights propensity score methods; the appendectomy cohort had a higher risk of sepsis than the non-appendectomy cohort. We further excluded individuals with a follow-up period of $<3$ months and analysed only new sepsis occurring at least 90 days after appendectomy. Patients with appendectomy had a significantly higher risk of developing sepsis than those without appendectomy (aHR, 1.86; 95\% CI, $1.61-2.14 ; \mathrm{P}<0.001)$. The Kaplan-Meier curves are shown in Fig. 1. The cumulative incidence of sepsis was lower in patients without appendectomy than in patients with appendectomy.

\section{Discussion}

In this large, population-based cohort study, we observed a 1.29-fold higher risk of sepsis in patients undergoing appendectomy, and it was especially higher in patients younger than 50 years (aHR: 1.58, 95\% CI, 1.50-1.68) and those within five years of follow-up after appendectomy. Diabetes was a comorbidity with a relatively higher adjusted hazard ratio (aHR: 2.01, 95\% CI, 1.94-2.09) for sepsis compared with other comorbidities. Furthermore, the cumulative incidence of sepsis among patients undergoing appendectomy significantly increased with time. These findings support our hypothesis that there is an association between prior appendectomy and the future risk of sepsis. To the best of our knowledge, this is the first and largest epidemiological study to use a nationwide 


\begin{tabular}{|c|c|c|c|c|c|c|c|c|}
\hline & \multicolumn{6}{|c|}{ Appendectomy } & \multirow{3}{*}{$\begin{array}{l}\text { Crude HR } \\
(95 \% \text { CI })\end{array}$} & \multirow{3}{*}{$\begin{array}{l}\text { Adjusted HR \& } \\
(95 \% \text { CI })\end{array}$} \\
\hline & \multicolumn{3}{|c|}{ No } & \multicolumn{3}{|l|}{ Yes } & & \\
\hline & Event & PY & Rate $^{\#}$ & Event & PY & Rate $^{\#}$ & & \\
\hline \multicolumn{9}{|l|}{ Age } \\
\hline $20-49$ & 1925 & $1,345,408$ & 1.43 & 3080 & $1,348,925$ & 2.28 & $1.60(1.51,1.69) * * *$ & $1.58(1.50,1.68) * * *$ \\
\hline $50-64$ & 1905 & 28,6471 & 6.65 & 2562 & 275538 & 9.30 & $1.40(1.32,1.49)^{* * *}$ & $1.40(1.32,1.48) * * *$ \\
\hline$\geq 65$ & 4848 & 176,826 & 27.4 & 5205 & 167,090 & 31.2 & $1.14(1.10,1.19)^{* * *}$ & $1.13(1.09,1.18)^{* * * *}$ \\
\hline \multicolumn{9}{|l|}{ Gender } \\
\hline Women & 3775 & 879,724 & 4.29 & 5076 & 872,819 & 5.82 & $1.36(1.30,1.41) * * *$ & $1.39(1.33,1.45)^{* * * *}$ \\
\hline Men & 4903 & 928,981 & 5.28 & 5771 & 918,734 & 6.28 & $1.19(1.15,1.24)^{* * *}$ & $1.22(1.17,1.27)^{* * *}$ \\
\hline \multicolumn{9}{|c|}{ Comorbidity $^{\S}$} \\
\hline No & 4258 & $1,617,489$ & 2.63 & 6137 & $1,605,213$ & 3.82 & $1.45(1.40,1.51)^{* * *}$ & $1.51(1.45,1.57)^{* * *}$ \\
\hline Yes & 4420 & 191,216 & 23.1 & 4710 & 186,340 & 25.3 & $1.09(1.05,1.14)^{* * *}$ & $1.10(1.06,1.15)$ *** \\
\hline \multicolumn{9}{|c|}{ Follow-up years } \\
\hline$<1$ & 1055 & 249,563 & 4.23 & 2073 & 248,222 & 8.35 & $1.97(1.83,2.13)^{* * *}$ & $1.98(1.84,2.14) * * *$ \\
\hline $1-4$ & 3682 & 820,155 & 4.49 & 4606 & 813,688 & 5.66 & $1.26(1.21,1.32)^{* * *}$ & $1.29(1.23,1.34)^{* * * *}$ \\
\hline$\geq 5$ & 3941 & 738,986 & 5.33 & 4168 & 729,643 & 5.71 & $1.07(1.03,1.12)^{* *}$ & $1.11(1.06,1.16)^{* *}$ \\
\hline
\end{tabular}

Table 3. Incidence and hazard ratio of sepsis for individuals with and without appendectomy. CI confidence interval; HR, hazard ratio; PY, person-years; "Incidence rate per 1,000 person-years; ${ }^{8}$ Multivariable analysis including age, gender and comorbidities of diabetes mellitus, hypertension, hyperlipidemia, atrial fibrillation, stroke and congestive heart failure; ' Individuals with any comorbidity of diabetes mellitus, hypertension, hyperlipidemia, atrial fibrillation, stroke and congestive heart failure were classified into the comorbidity group; $* * \mathrm{P}<0.01, * * * \mathrm{P}<0.001$.

\begin{tabular}{|l|l|l|}
\hline Variable & $\begin{array}{l}\text { Crude HR } \\
(\mathbf{9 5 \%} \mathbf{C I})\end{array}$ & $\begin{array}{l}\text { Adjusted HR \& } \\
(\mathbf{9 5 \%} \mathrm{CI})\end{array}$ \\
\hline $\begin{array}{l}\text { Inverse probability } \\
\text { of treatment } \\
\text { weights }\end{array}$ & $1.26(1.24,1.29) * * *$ & $1.31(1.28,1.33) * * *$ \\
\hline $\begin{array}{l}\text { Excluded due to a } \\
\text { follow-up period } \\
\text { of }<3 \text { months }\end{array}$ & $2.03(1.76,2.33) * * *$ & $1.86(1.61,2.14) * * *$ \\
\hline
\end{tabular}

Table 4. Sensitivity analyses of sepsis risk for individuals with and without appendectomy using inverse probability of treatment weights propensity score methods and individuals excluded due to a follow-up period of $<3$ months. CI, confidence interval; HR, hazard ratio; PY, person-years; ${ }^{\circledR}$ Multivariable analysis including age, gender and comorbidities of diabetes mellitus, hypertension, hyperlipidemia, atrial fibrillation, stroke and congestive heart failure; $* * * \mathrm{P}<0.001$.

longitudinal dataset to identify an increased sepsis risk among patients with appendectomy. These results highlight the possibility that the absence of a vermiform appendix might provoke an increased risk of sepsis.

The pathophysiology underlying the relationship between an appendectomy and subsequent sepsis remains uncertain. The appendix is the primary site of production of secretory immunoglobulin $\mathrm{A}^{15}$, which binds to pathogenic bacteria with high affinity and promotes their elimination ${ }^{16}$. Nevertheless, it also binds to the commensal gut flora with low affinity and plays a crucial role in host protection. Therefore, the appendix plays an important role in regulating the size and composition of the gut microbiota ${ }^{16}$. The function of the appendix is to act as a reservoir of commensal flora to rapidly re-inoculate the gut through biofilm regeneration and shedding following enteric infections or antibiotics administration ${ }^{16-18}$. We speculate that the lack of an appendix could influence the restoration of the gut microbiome, which could increase susceptibility to sepsis and related organ dysfunction. The gut has been considered a motor of sepsis and multiple organ dysfunction syndrome for the past one third century ${ }^{19}$. It is hypothesised that the intestinal epithelium becomes highly permeable and apoptotic during critical illness, which results in intestinal flora leaking into the systemic circulation and potentially leading to systemic inflammation and organ failure. Also, various pre-clinical studies have revealed the existence of so-called 'gut-organ axes', such as the gut-brain axis and the gut-lung axis ${ }^{14}$. In addition to cytokines, communication on these axes might be mediated by microbe-associated molecular patterns, such as peptidoglycan, LPS, flagellin and microbiome-derived metabolites, which can be transferred from the gut to the systemic circulation ${ }^{14}$. These microbe-associated molecular patterns have the potential to modulate immune cells to enhance the systemic inflammatory response. The appendix also has the highest concentration of mucosa-associated lymphoid tissue and is capable of responding to various pathogens and microbial antigens present in the gut. Removal of the appendix, which is an immune organ and a reservoir of beneficial flora, might lead to local and distant insults through changes in defence mechanisms and disruption of the equilibrium regulated by complex crosstalk 


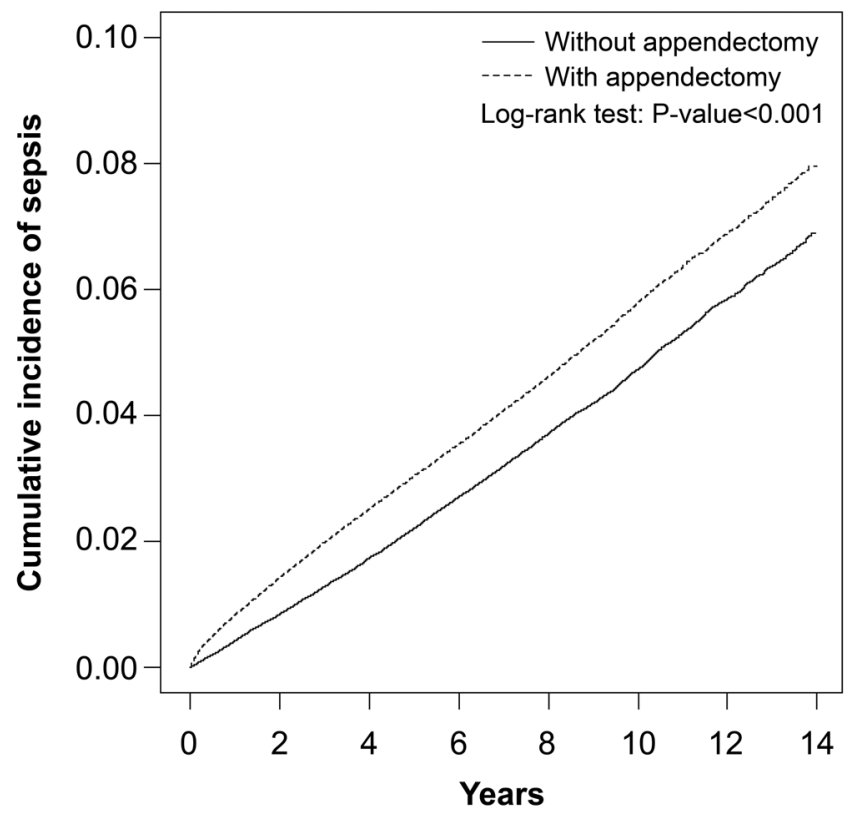

Figure 1. Cummulative incidence of sepsis between individuals with and without appendectomy.

between the gut, mucosal immunity and the microbiota. This process could lead to the progression and pathogenesis of sepsis and organ dysfunction in susceptible populations. For instance, cancer patients who must receive cytotoxic chemotherapies and subsequently suffer intestinal mucosal disturbance, are particularly at risk of sepsis.

The second finding of this study was that appendectomy led to a significantly higher risk of sepsis in the middle aged (20-49 years) patients (aHR 1.58, 95\% CI, 1.50-1.68) than in those with prior appendectomy at $>65$ years (aHR 1.13, 95\% CI, 1.09, 1.18). In elderly individuals, the function of the appendix might be attenuated, given the length/diameter and the number of lymphoid follicles of the human vermiform appendix degenerate with age ${ }^{20}$. Therefore, appendectomy might be less likely to affect their immune defences. Another finding of our study was that diabetes was a comorbidity with a relatively higher adjusted hazard ratio (aHR: $2.01,95 \% \mathrm{CI}$, 1.94-2.09) compared with other comorbidities. We speculate that appendectomy may worsen pre-existing dysbiosis in patients with diabetes mellitus and thus increased the risk of sepsis.

In addition, sepsis might be induced by complications of recent appendicitis and subsequent surgical procedure, such as intra-abdominal abscess, surgical site infection and intestinal obstruction. We also found that there was a relatively higher risk of sepsis (aHR: 1.98, 95\% CI, 1.84-2.14) within the first years of follow-up after undergoing appendectomy. However, the risk of sepsis was still significantly higher in the follow-up periods of 1-4 and $\geq 5$ years. One recent study by Ninh et al. ${ }^{21}$ reported that 311 patients $(0.43 \%)$ among 72,538 participants developed post-appendectomy related sepsis, and open appendectomy increased the risk of sepsis. We further analysed only new sepsis occurring at least 90 days after appendectomy and found that patients with appendectomy had a significantly higher future risk of sepsis than those without appendectomy. There is increasing evidence to show that non-surgical management with antibiotics might be an effective and safe treatment for acute uncomplicated appendicitis in children and adults ${ }^{17,22,23}$. Our study provides evidence of a correlation between previous appendectomy and subsequent sepsis risk. Therefore, we recommend thoughtful consideration before performing incidental or prophylactic appendectomy. In other words, the potential benefit of appendectomy must be weighed judiciously after considering the patient's personal risk-benefit profile. In addition, probiotics that can modulate the function of the intestinal immune system might have a beneficial impact on those undergoing appendectomy; further dedicated research on this issue is warranted.

The major strengths of this study were the large sample size and the relatively long duration of the follow-up, in which a complete history of medical service use was available for all cases and controls. Therefore, our study design minimised selection, information and recall bias, which made testing our hypothesis feasible. Furthermore, we used strict exclusion criteria and propensity score matching to control for potential confounders. Nonetheless, there are several limitations to be noted. First, the NHIRD does not disclose information regarding the patients' diet, socioeconomic status, family history, personal lifestyle, psychologic factors, body mass index and microbiomes of the study populations, which might be associated risk factors for the development of sepsis. Although we adjusted for various comorbidities and matched propensity scores, these unmeasured confounding factors might have affected our results. Second, accurately quantifying the incidence of sepsis is difficult. Because there is no definitive tissue or serological test for sepsis, the gold standard for sepsis diagnosis is clinical identification of life-threatening organ dysfunction caused by infection ${ }^{2}$. At the nationwide population level, this would require either a prospective cohort study or retrospective medical record review, the scale of which is unrealistic for routine disease surveillance. Therefore, routinely collected data are analysed to estimate the incidence of sepsis, mainly based on ICD coding of cases. However, sepsis patients comprise heterogeneous groups and are categorised with different diagnostic administrative codes. We focused on patients with diagnoses of sepsis 
(ICD-9-CM code 038.x, 003.1 and 036.2). The definition of sepsis was based on ICD-9-CM codes, rather than clinical diagnostic criteria such as the sepsis-3 definition ${ }^{2}$. This is an inherent limitation of population-based datasets such as the NHIRD. Although the accuracy of these codes has been validated ${ }^{24-31}$, Valentine et al. reported that the sensitivity of coding data regarding sepsis was $78 \%$ and $64 \%$ of intensive care unit (ICU) and non-ICU cases using ICD-10 codes at admission level ${ }^{32}$. Another study by Fleischmann-Struzek et al. also indicated that explicit sepsis coding strategies may underestimate sepsis incidence and implicit strategies (an infection code plus a code indicting organ dysfunction) might overestimate the incidence of sepsis ${ }^{33}$. Thus, potential misclassification is worthy of attention; however, we believe that any misclassification is non-differential for exposed and unexposed cohorts and that the undetected presence of an infectious code plus organ dysfunction would be more likely to lead to underestimation of the associations examined. Moreover, clinical judgement might vary between physicians, so diagnoses could also vary and affect their validity. However, the NHI in Taiwan has established an ad hoc committee to monitor the accuracy of the claim data to prevent violations. Third, due to the potential for residual confounding inherent in database research, the results should be interpreted with caution. Randomised controlled trials to demonstrate the effect of appendectomy and subsequent sepsis are laborious and resource intensive. Such a study is difficult to conduct due to ethical issues in randomising patients to undergo appendectomy to observe adverse outcomes. Finally, it remains uncertain whether the finding in our study can be extrapolated to other ethnic groups, as the majority of our patients were Chinese. Clinical studies should be conducted in patients from other countries and must include people of different ethnicities to further elucidate the associations.

\section{Conclusion}

Patients requiring appendectomy had a 1.29 times greater risk of developing sepsis than those not undergoing appendectomy. The cumulative incidence of sepsis among patients receiving appendectomy significantly increased over time. Future basic and clinic research is needed to clarify the pathogeneses underlying these associations.

\section{Methods}

Data source. This study employed the NHIRD, which consists of claim data after removing identifying information. The database was established in 1995 and includes records from over $99 \%$ of insured people who have resided in Taiwan. Diagnostic codes were defined based on the International Classification of Diseases, $9^{\text {th }}$ Revision, Clinical Modification (ICD-9-CM) codes. The Institutional Review Board of Research Ethics Committee II of China Medical University and Hospital [Approval number: CMUH104-REC2-115(CR-4)] has approved this study. Written consent from study subjects was not required and waived by the Institutional Review Board of Research Ethics Committee II of China Medical University and Hospital, because the NHIRD comprises de-identified data for research purposes. The study carried out in accordance with principles of Declaration of Helsinki.

Study population. Patients aged $>20$ years who underwent appendectomy (ICD-9-CM Procedure Code: 47.0 and 47.1) were assigned to the case cohort. The index date was the date patients underwent the appendectomy. Participants who did not undergo appendectomy were assigned to the comparison cohort. The study excluded patients with a history of sepsis (ICD-9-CM: 003.1, 036.2 and 038.x) before the index date, those who underwent appendectomy before the year 2000 and those who lacked complete information in the NHIRD. The exposed cohort and the unexposed cohort were matched using propensity score matching at a 1:1 ratio by the index year, sex, age and comorbidities. The main outcome was a new-onset hospitalised diagnosis of sepsis (ICD9-CM: 003.1, 036.2 and 038.x). All participants were observed until the end of 2013 or until their records were censored for death, emigration or discontinuation of enrolment in the NHIRD.

Comorbidities. The baseline history of comorbidity comprised diabetes mellitus (ICD-9-CM: 250 and A181), hypertension (ICD-9-CM: 401-405, A260 and A269), hyperlipidemia (ICD-9-CM: 272 and A182), atrial fibrillation (ICD-9-CM: 427.31), stroke (ICD-9-CM: 430-438 and A290-A299) and congestive heart failure (ICD-9-CM: 428).

Statistical analysis. The distributions of categorical demographic variables and comorbidities were compared between the exposed cohort and the unexposed cohort and the differences were examined using chi-squared tests. The median age of both cohorts was measured and tested using Mann-Whitney U test. The proportional hazards model assumption was also examined using a test of scaled Schoenfeld residuals. In the model evaluating the sepsis risk throughout the overall follow-up period, the results of the test revealed a significant relationship between the Schoenfeld residuals for appendectomy and follow-up time, suggesting that the proportionality assumption was violated $(\mathrm{P}<0.001)$. Cox proportional hazard models were employed to assess the risk of developing sepsis associated with appendectomy after adjusting for the following covariates: age, gender and comorbidities of diabetes mellitus, hypertension, hyperlipidemia, atrial fibrillation, stroke and congestive heart failure. HRs with $95 \%$ confidence intervals (95\% CIs) were calculated using this model.

Sub-analyses stratified by sex, age group, comorbidity and years of follow-up were also performed to assess the association between appendectomy and the subsequent risk of sepsis. Cumulative incidence rates and curves of sepsis were estimated and plotted by the Kaplan-Meier method, and log-rank tests were used to compare differences in time-to-event distributions between the case cohort and the comparison cohort. The two-tailed significance levels of all tests were set at 0.05. All data were analysed using SAS 9.4 software (SAS Institute Inc., Cary, NC, USA) and cumulative incidence curves were plotted in R software. 


\section{Data availability}

The dataset used in this study is held by the Taiwan Ministry of Health and Welfare (MOHW). The Ministry of Health and Welfare must approve our application to access this data, and patient consent is exempted due to de-identification of the NHIRD (Database NHIR, Taiwan). Available online: http://nhird.nhri.org.tw/en/index. $\mathrm{htm}$. All methods were performed in accordance with the relevant guidelines and regulations. Any researcher interested in accessing this dataset can submit an application form to the Ministry of Health and Welfare requesting access. Please contact the staff of MOHW (Email: stcarolwu@mohw.gov.tw) for further assistance. Taiwan Ministry of Health and Welfare Address: No.488, Sec. 6, Zhongxiao E. Rd., Nangang Dist., Taipei City 115, Taiwan (R.O.C.). Phone: $+886-2-8590-6848$. All relevant data are within the paper.

Received: 22 January 2020; Accepted: 21 May 2020;

Published online: 23 June 2020

\section{References}

1. van der Poll, T., van de Veerdonk, F. L., Scicluna, B. P. \& Netea, M. G. The immunopathology of sepsis and potential therapeutic targets. Nat. Rev. Immunol. 17, 407-420 (2017).

2. Singer, M. et al. The third international consensus definitions for sepsis and septic shock (Sepsis-3). JAMA 315, 801-810 (2016).

3. Gebbers, J. O. \& Laissue, J. A. Bacterial translocation in the normal human appendix parallels the development of the local immune system. Ann. N. Y. Acad. Sci. 1029, 337-343 (2004).

4. Im, G. Y. et al. The appendix may protect against Clostridium difficile recurrence. Clin. Gastroenterol. Hepatol. 9, 1072-1077 (2011).

5. Lauritano, E. C. et al. Small intestinal bacterial overgrowth recurrence after antibiotic therapy. Am. J. Gastroenterol. 103, 2031-2035 (2008).

6. Chung, W. S., Lin, C. L. \& Hsu, C. Y. Women who had appendectomy have increased risk of systemic lupus erythematosus: a nationwide cohort study. Clin. Rheumatol. 37, 3009-3016 (2018).

7. Tzeng, Y. M. et al. An appendectomy increases the risk of rheumatoid arthritis: a five-year follow-up study. PLoS One 10, e0126816 (2015).

8. Burke, K. E., Boumitri, C. \& Ananthakrishnan, A. N. Modifiable environmental factors in inflammatory bowel disease. Curr. Gastroenterol. Rep. 19, 21-21 (2017).

9. Cope, J. U. et al. Appendectomy during childhood and adolescence and the subsequent risk of cancer in Sweden. Pediatrics 111, 1343-1350 (2003).

10. Mohammadi, M. et al. Risk of lymphoid neoplasms in a Swedish population-based cohort of 337,437 patients undergoing appendectomy. Scand. J. Gastroenterol. 51, 583-589 (2016).

11. Wu, S. C. et al. Association between appendectomy and subsequent colorectal cancer development: an Asian population study. PLoS One 10, e0118411 (2015).

12. Rasmussen, T., Fonnes, S. \& Rosenberg, J. Long-term complications of appendectomy: A systematic review. Scand. J. Surg. 107, $189-196(2018)$

13. Haak, B. W. \& Wiersinga, W. J. The role of the gut microbiota in sepsis. Lancet Gastroenterol. Hepatol. 2, 135-143 (2017).

14. Haak, B. W., Prescott, H. C. \& Wiersinga, W. J. Therapeutic potential of the gut microbiota in the prevention and treatment of sepsis. Front. Immunol. 9, 2042 (2018).

15. Masahata, K. et al. Generation of colonic IgA-secreting cells in the caecal patch. Nat. Commun. 5, 3704 (2014).

16. Girard-Madoux, M. J. H. et al. The immunological functions of the Appendix: an example of redundancy? Semin. Immunol. 36, 31-44 (2018).

17. Hall, N. J. \& Eaton, S. Non-operative management of appendicitis in children. Arch. Dis. Child. 103, 498-502 (2018).

18. Vitetta, L., Vitetta, G. \& Hall, S. The brain-intestinal mucosa-appendix- microbiome-brain loop. Diseases 6 (2018).

19. Klingensmith, N. J. \& Coopersmith, C. M. The gut as the motor of multiple organ dysfunction in critical illness. Crit. Care Clin. 32, 203-212 (2016).

20. Sarkar, A. et al. A glimpse towards the vestigiality and fate of human vermiform appendix-a histomorphometric study. J. Clin. Diagn. Res 9, AC11-AC15 (2015).

21. Ninh, A., Wood, K., Bui, A. H. \& Leitman, I. M. Risk factors and outcomes for sepsis after appendectomy in adults. Surg. Infect. (Larchmt) 20, 601-606 (2019).

22. Sallinen, V. et al. Meta-analysis of antibiotics versus appendicectomy for non-perforated acute appendicitis. Br. J. Surg. 103, 656-667 (2016).

23. Salminen, P. et al. Five-year follow-up of antibiotic therapy for uncomplicated acute appendicitis in the APPAC randomized clinical trial. JAMA 320, 1259-1265 (2018).

24. Chao, P. W. et al. Association of postdischarge rehabilitation with mortality in intensive care unit survivors of sepsis. Am. J. Respir. Crit. Care. Med. 190, 1003-1011 (2014).

25. Chou, C. H. et al. Septicemia is associated with increased risk for dementia: a population-based longitudinal study. Oncotarget 8 , 84300-84308 (2017).

26. Hsieh, M. S. et al. Hospital outcomes and cumulative burden from complications in type 2 diabetic sepsis patients: a cohort study using administrative and hospital-based databases. Ther. Adv. Endocrinol. Metab. 10, 2042018819875406 (2019).

27. Kao, L. T., Sheu, J. J., Lin, H. C., Tsai, M. C. \& Chung, S. D. Association between sepsis and dementia. J. Clin. Neurosci. 22, 1430-1433 (2015).

28. Lee, C. W., Chen, H. J., Liang, J. A. \& Kao, C. H. Risk of sepsis in patients with amyotrophic lateral sclerosis: a population-based retrospective cohort study in Taiwan. BMJ Open 7, e013761 (2017).

29. Ou, S. M. et al. Long-term mortality and major adverse cardiovascular events in sepsis survivors. A nationwide population-based study. Am. J. Respir. Crit. Care. Med. 194, 209-217 (2016).

30. Shih, C. J., Chao, P. W., Ou, S. M. \& Chen, Y. T. Long-term risk of cardiovascular events in patients with chronic kidney disease who have survived sepsis: a nationwide cohort study. J. Am. Heart Assoc. 6, e004613 (2017).

31. Shih, C. J. et al. Association between use of oral anti-diabetic drugs and the risk of sepsis: a nested case-control study. Sci. Rep. 5, 15260 (2015)

32. Valentine, J. C., Haeusler, G., Worth, L. \& Thursky, K. Sepsis incidence and mortality are underestimated in Australian intensive care unit administrative data. Med. J. Aust. 210, 188-188.e181 (2019).

33. Fleischmann-Struzek, C. et al. Comparing the validity of different ICD coding abstraction strategies for sepsis case identification in German claims data. PLoS One 13, e0198847 (2018).

\section{Acknowledgements}

This work was supported by grants from the Ministry of Health and Welfare, Taiwan (MOHW108TDU-B-212-133004), China Medical University Hospital, Academia Sinica Stroke Biosignature Project (BM10701010021), MOST Clinical Trial Consortium for Stroke (MOST 108-2321-B-039-003-), Taichung Veterans General Hospital (TCVGH-1096505B), Tseng-Lien Lin Foundation, Taichung, Taiwan and Katsuzo and Kiyo Aoshima Memorial Funds, Japan. 


\section{Author contributions}

All authors contributed to the conception, design and interpretation of the work substantially. Drafting of the manuscript was done by M.C.W., H.K.T. and C.L.L. The integrity of the data was supervised by J.C.C.W. C.L.L. contributed to research data and data analysis. J.C.C.W. revised the manuscript critically. All authors approved the final version of the manuscript.

\section{Competing interests}

The authors declare no competing interests.

\section{Additional information}

Correspondence and requests for materials should be addressed to J.C.-C.W.

Reprints and permissions information is available at www.nature.com/reprints.

Publisher's note Springer Nature remains neutral with regard to jurisdictional claims in published maps and institutional affiliations.

(c) (i) Open Access This article is licensed under a Creative Commons Attribution 4.0 International License, which permits use, sharing, adaptation, distribution and reproduction in any medium or format, as long as you give appropriate credit to the original author(s) and the source, provide a link to the Creative Commons license, and indicate if changes were made. The images or other third party material in this article are included in the article's Creative Commons license, unless indicated otherwise in a credit line to the material. If material is not included in the article's Creative Commons license and your intended use is not permitted by statutory regulation or exceeds the permitted use, you will need to obtain permission directly from the copyright holder. To view a copy of this license, visit http://creativecommons.org/licenses/by/4.0/.

(c) The Author(s) 2020 